\title{
Preserved verbal memory function in left medial temporal pathology involves reorganisation of function to right medial temporal lobe
}

\author{
Mark P. Richardson, ${ }^{\mathrm{a}, *}$ Bryan A. Strange, ${ }^{\mathrm{b}}$ John S. Duncan, ${ }^{\mathrm{a}}$ and Raymond J. Dolan ${ }^{\mathrm{b}}$ \\ ${ }^{a}$ Department of Clinical and Experimental Epilepsy, Institute of Neurology, Queen Square, London WC1N 3BG, UK \\ ${ }^{\mathrm{b}}$ Wellcome Department of Imaging Neuroscience, Institute of Neurology, Queen Square, London WC1N 3BG, UK
}

\begin{abstract}
The left hippocampus and related structures mediate verbal memory function. The mechanism underlying preserved verbal memory function in patients with left hippocampal damage is unknown. Temporal lobe epilepsy, a common disease, is frequently the consequence of a characteristic hippocampal pathology termed hippocampal sclerosis, which may also affect the amygdala. In this setting, mapping the sites of memory function is a vital component of planning for surgical treatment for epilepsy. Using event-related functional magnetic resonance imaging, we studied 24 right-handed nonamnesic patients with left hippocampal sclerosis and 12 normal controls, performing a verbal encoding task. The patients were subdivided into two groups according to presence or absence of additional left amygdala pathology. Analysis of the data employed a two-level random-effects design, examining the main effects of subsequent memory in each group, as well as the differences between the groups. Additional effects of emotionality of the remembered words were also examined. Verbal memory encoding involved activation of left hippocampus in normals, but was associated with reorganisation to right hippocampus and parahippocampal gyrus in the patients. The additional presence of left amygdala sclerosis resulted in reorganisation for encoding of emotional verbal material to right amygdala. Retained verbal memory function in the presence of left medial temporal lobe pathology is mediated by recruitment of a parallel system in the right hemisphere consistent with adaptive functional reorganisation. The findings indicate a high degree of plasticity in medial temporal lobe structures.
\end{abstract}

(C) 2003 Elsevier Inc. All rights reserved.

Keywords: Epilepsy; Memory; Hippocampus; Reorganisation

\section{Introduction}

The human hippocampus and related medial temporal lobe (MTL) structures are crucial to long-term episodic memory function (Squire, 1992). Studies of subjects with injury to this area reveal a characteristic syndrome of amnesia (Squire and Zola-Morgan, 1991). The role of the hippocampus has been confirmed and extended by functional imaging in normal subjects which reveal hippocampal activity in long-term memory tasks (Lepage et al., 1998; Cabeza and Nyberg, 2000). A dissociation between left hippocampus, subserving verbal memory, and right hippocampus, mediating visual memory, is evident from stud-

\footnotetext{
* Corresponding author: National Hospital for Neurology and Neurosurgery, Box 29, Queen Square, London WC1N 3BG, UK. Fax: +44-207837-3941.

E-mail address: m.richardson@ion.ucl.ac.uk (M.P. Richardson).
}

ies of patients (Smith and Milner, 1981; Frisk and Milner, 1990) and functional neuroimaging in normal subjects (Kelley et al., 1998).

The hippocampus is the site of a common pathology, hippocampal sclerosis (HS), which frequently underlies a common disease, temporal lobe epilepsy (TLE). Although patients with TLE may have memory impairment, amnesia is unusual (Helmstaedter and Kurthen, 2001). HS is detected in vivo using magnetic resonance imaging (MRI) (Duncan, 1997) and its hallmarks are reduced hippocampal volume and increased T2 signal, with other medial temporal structures and more remote brain regions generally showing no pathology (Sisodiya et al., 1997). Although bilateral HS is found in a minority, standardised imaging criteria used in this study allow unilateral HS to be identified (Woermann et al., 1998). Therefore, subjects with HS provide a unique opportunity to study long-term consequences of isolated unilateral hippocampal injury. 
Given the role of left hippocampus in verbal memory function, the observation that verbal episodic memory is relatively intact in subjects with left HS suggests reorganisation of episodic memory processes to other brain loci. During evaluation for neurosurgical treatment of epilepsy, TLE patients may undergo a procedure in which first one hemisphere, then the other, is anaesthetised by a shortacting barbiturate injected into the internal carotid artery (Wada test), allowing the memory capacity of each unanaesthetised hemisphere to be tested in isolation. Usually, memory is superior in the hemisphere unaffected by HS. Bilateral temporal lobe resection in TLE patients produces devastating amnesia, as in the famous amnesic patient HM (Scoville and Milner, 1957). This implies that in unilateral HS, memory function is reallocated from the hippocampus affected by HS to the contralateral temporal lobe.

Anterior to the hippocampus is the amygdala, a region linked to emotional perception and memory (Dolan, 2002). A close functional relationship between amygdala and hippocampus is suggested by enhancement in free recall of emotionally significant relative to neutral material (Phelps et al., 1997). Patients with isolated amygdala damage show a specific impairment in recall of emotional stimuli (Adolphs et al., 1997). Functional imaging studies show that amygdala activity correlates with subsequent memory for emotional material (Hamann et al., 1999; Strange et al., 2000). In patients with HS, the amygdala may be affected by the same sclerotic pathology as the hippocampus, although the severity of hippocampal and amygdala sclerosis in a given patient may be uncorrelated (Hudson et al., 1993). As in the case of the hippocampus, amygdala pathology may be assessed using MRI by measuring T2 signal (Van Paesschen et al., 1996; Bartlett et al., 2002).

Functional imaging studies have frequently sought evidence of the functional adaptation of the brain to the presence of a lesion in the brain or peripheral nervous system. Few instances have emerged in which good task performance is associated with a pattern of brain activity clearly different from that in normal subjects. Following stroke, clinical recovery is related to functional recovery within the damaged area itself, with reorganisation of function to remote brain regions (such as the opposite undamaged hemisphere) being associated with poor recovery (Warburton et al., 1999; Pineiro et al., 2001; Johansen-Berg et al., 2002). Reorganisation of brain function resulting in good performance has been demonstrated for sensory functions as evidenced in long-term deaf (Neville et al., 1998) or blind (Sadato et al., 1996) adults. Reorganisation of language to right frontal regions has been reported in recovered aphasics with left frontal lesions (Blasi et al., 2002), in addition to reorganisation of motor function to the ipsilateral hemisphere in adults with congenital hemiparesis (Staudt et al., 2002). The ability of regions outside the left MTL to subserve residual verbal memory functions has not been demonstrated.
In this study, we describe a functional MRI (fMRI) study of verbal encoding in subjects with an MRI-based diagnosis of unilateral left HS, compared to normal controls. We hypothesised that pathology in left medial temporal lobe structures would result in reallocation of verbal encoding functions to the right MTL. As functional neuroimaging studies of patients may be confounded by heterogeneity of sample and poor task performance compared to normals, we studied a homogeneous group of right-handed patients with identical pathology and compared encoding event types which showed the least variance between patients and normals.

\section{Materials and methods}

\section{Subjects}

We studied 12 right-handed normal volunteers and 24 right-handed patients recruited from the National Hospital for Neurology and Neurosurgery, London. All lived in southern England and were fluent English speakers. All patients had active temporal lobe epilepsy and had undergone MRI at 1.5 T (Horizon Echospeed, General Electric, Milwaukee, WI, USA) (Duncan et al., 1996; Duncan, 1997; Woermann et al., 1998), which had identified left HS and normal right hippocampus. The patients were divided into two groups based on left amygdala T2: the first group (HS) had normal left amygdala T2; the second group had abnormally elevated left amygdala T2 (HSAS) (Bartlett et al., 2002). The age range of the patient group was 18-54 (median 29) and of the normal control group was 19-48 (median 31); 10 patients and 8 normal subjects were male; all patients were on anticonvulsant medication. All patients had undergone neuropsychometry and had a verbal IQ $>80$ (range 80-124, median 102). IQ data were not obtained for the normal subjects. Approval for this study was obtained from the joint ethics committee of the hospital and the Institute of Neurology. All subjects gave written informed consent.

\section{Imaging}

Subjects were scanned at $2 \mathrm{~T}$ (Siemens Vision, Siemens, Erlangen, Germany), acquiring gradient-echo echo-planar T2*-weighted image volumes, providing blood oxygenation level-dependent contrast, with 33 contiguous slices covering the whole brain, voxel dimensions $3 \times 3 \times 3.67 \mathrm{~mm}$, TE $40 \mathrm{~ms}$, and TR $2.5 \mathrm{~s}$. SPM99 was used for image analysis (Friston et al., 1995). The images were realigned and corrected for slice timing differences. All of the images from each subject were then transformed to the standard anatomical volume and smoothed with an 8-mm kernel.

\section{Psychological task}

During scanning subjects were presented 255 single words previously used elsewhere (Otten et al., 2001), including 36 emotionally aversive words (e.g., "cancer," 

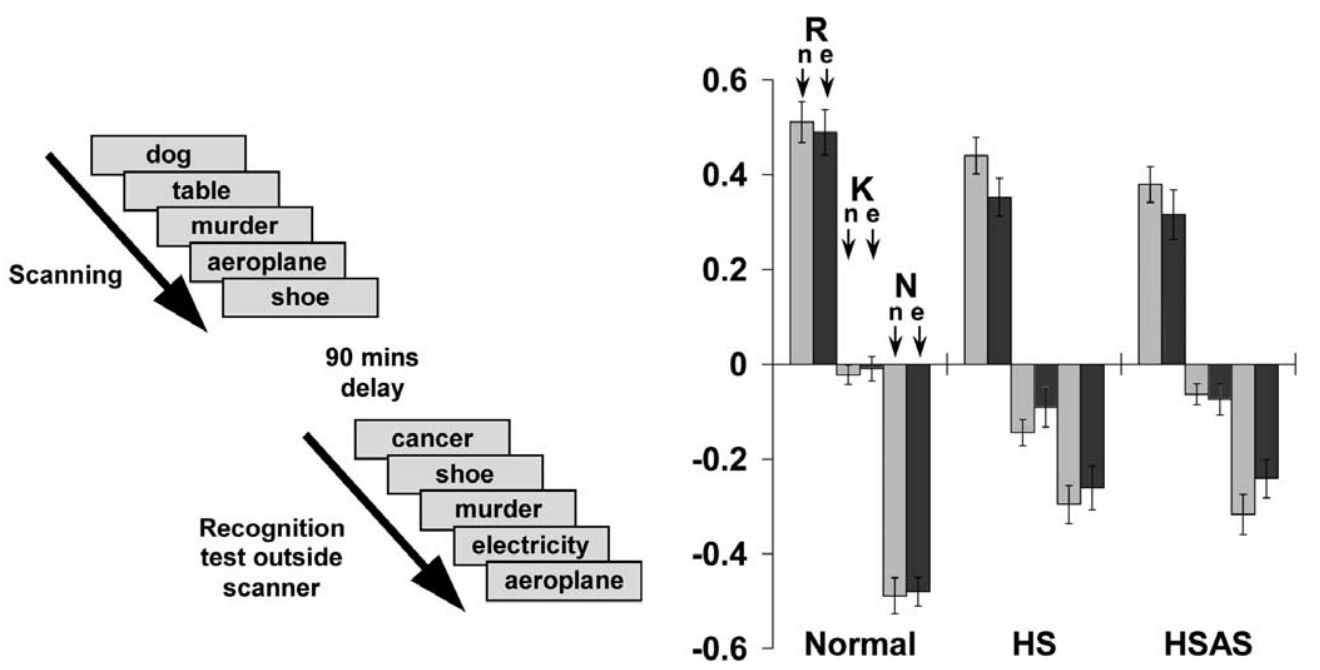

Fig. 1. Psychological task and behavioural data. Left: Psychological task summarised. Right: Recognition accuracy for each stimulus type and each group. In this and all subsequent figures, dark gray bars represent responses to emotional items, light gray bars show responses to neutral items, error bar \pm 1 SEM. $\mathrm{R}, \mathrm{K}$, and $\mathrm{N}$ responses as defined in the method. HS: hippocampal sclerosis alone. HSAS: hippocampal and amygdala sclerosis.

"rape," "terrorist") (Strange et al., 2000), 1 every 4.5 s. Subjects were instructed to indicate with a single right-hand button press whether the word indicated a living or nonliving entity. Ninety minutes after scanning, subjects performed a surprise recognition memory test outside fMRI scanning (Fig. 1). At recognition, single words were presented every $4.5 \mathrm{~s}$ in a manner identical to that used in scanning: 255 words presented during scanning, randomly mixed with 170 neutral foils and 36 emotional foils. For each stimulus, subjects were asked to indicate with a righthand button press if they could remember seeing the word on the screen during scanning ( $\mathrm{R}$ response), if the word seemed familiar (K response), or if the word was new ( $\mathrm{N}$ response) (Tulving, 1985). The encoding stimuli were then conditionalised according to these recognition responses; seven event types were identified: correct $\mathrm{R}$ responses for neutral stimuli $(\mathrm{Rn})$, correct $\mathrm{K}$ responses to neutral stimuli $(\mathrm{Kn})$, incorrect $\mathrm{N}$ responses to neutral stimuli (forgotten, $\mathrm{Fn}$ ), and similarly for emotional stimuli ( $\mathrm{Re}, \mathrm{Ke}, \mathrm{Fe})$. A single category for all encoding task errors or missed responses was included, hence in total seven event types were indicated. To calculate recognition accuracy for each event type ( $\mathrm{Rn}, \mathrm{Kn}, \mathrm{Re}, \mathrm{Ke})$, stimuli seen in the recognition test were classified as correct identifications (hits) and incorrect responses to foils (false alarms). Recognition accuracy was calculated for each of these four event types as (hit rate) (false alarm rate). To calculate the recognition accuracy for $\mathrm{N}$ responses, stimuli in the recognition test were similarly classified as correct rejections and misses; recognition accuracy for $\mathrm{N}$ responses was calculated as (miss rate) (correct rejection rate), for neutral and emotional stimuli separately. The highest possible score was +1 , indicating perfect identification of stimuli present at encoding; the lowest score, -1 , indicated perfect identification of foils.

\section{Imaging analysis}

To test for subsequent memory effects, imaging data were analysed with a two-level random-effects analysis employing an event-related design (Friston et al., 1998). At the first level, trial-specific responses were modelled for each subject by convolving a delta function with the canonical haemodynamic response function (HRF) to create regressors of interest, one regressor for each of the seven event types described above. Each subject's movement parameters were included as confounds. A high-pass filter of $120 \mathrm{~s}$ was used and data were scaled for global activity. Parameter estimates pertaining to the height of the HRF for each regressor were calculated at each voxel. Contrasts of parameter estimates were calculated to produce four "contrast images" for each subject ( $\mathrm{Rn}$ minus $\mathrm{Kn}, \mathrm{Re}$ minus Ke, main effect of $\mathrm{R}$ minus $\mathrm{K}$, interaction between $\mathrm{R}$ minus $\mathrm{K}$ and emotion). These images were used for the second-level analyses.

At the second level, one-sample $T$ tests were used to examine effects within groups, and two-sample $T$ tests used to examine between-group effects. We chose $p<0.05$ corrected (within the small volume of the MTL using an image mask) as the threshold for significance. We report all MTL activations that survived this threshold and report descriptively MTL regions showing uncorrected significance where we replicate previous findings or showing a nonsignificant trend where this contributes to interpretation of the data.

\section{Results}

Left and right hippocampal volumes were significantly different in the patients: mean left hippocampal volume $1834 \mathrm{~mm}^{3}$, mean right hippocampal volume $2797 \mathrm{~mm}^{3}$ 
Table 1

Activation peaks for subsequent memory effects in medial temporal lobe

\begin{tabular}{|c|c|c|c|c|}
\hline Contrast & Fig. & MNI coordinates & $Z$ score & Region \\
\hline Main effect of memory, normal controls & $2 \mathrm{a}$ & $-32-18-18$ & 2.35 & Left hippocampus \\
\hline \multirow[t]{4}{*}{ Main effect of memory, patients } & $2 b$ & $18-6-20$ & $4.29 *$ & Right amygdala \\
\hline & $2 \mathrm{c}$ & $26-14-18$ & $3.53^{*}$ & Right hippocampus \\
\hline & $2 \mathrm{~d}$ & $24-28-20$ & $3.27 *$ & Right parahippocampal gyrus \\
\hline & $2 \mathrm{e}$ & $-26-24-20$ & $3.32 *$ & Inferior to left hippocampus \\
\hline Two-way interaction between memory and group (collapsed across emotionality) & $3 a$ & $26-20-22$ & $3.45^{*}$ & Right parahippocampal gyrus \\
\hline \multirow[t]{2}{*}{ Two-way interaction between memory and emotionality (collapsed across group) } & $3 b$ & $-28-22-10$ & $3.0^{*}$ & Left hippocampus \\
\hline & $3 \mathrm{c}$ & $30-18-14$ & 2.71 & Right hippocampus \\
\hline \multirow[t]{2}{*}{ Three-way interaction between memory, emotionality, and group } & $4 \mathrm{a}$ & $8-22$ & $3.77 *$ & Right periamygdala \\
\hline & $4 \mathrm{~b}$ & $26-10-14$ & $3.0^{*}$ & Right amygdala/hippocampus \\
\hline
\end{tabular}

For each effect, the Montreal Neurological Institute (MNI) coordinates, $Z$ score, anatomical location, and relevant figure are given.

* Regions significant at $p<0.05$ corrected.

(paired $T$ test, $p<0.001$, two-tailed). The patients were divided into two groups based on left amygdala T2: the first group (HS) had normal left amygdala T2; the second group (HSAS) had abnormally elevated left amygdala T2 (Bartlett et al., 2002) (mean relaxation time $88.2 \mathrm{~ms}$ in HS group versus $94.3 \mathrm{~ms}$ in HSAS group; $T$ test, $p<0.001$, twotailed). There was a strong trend for HSAS patients to have larger left hippocampi than HS patients (HS group left hippocampus mean $1984 \mathrm{~mm}^{3}$ versus mean left hippocampus $1683 \mathrm{~mm}^{3}$ in HSAS group; $T$ test, $p=0.056$, twotailed). Hence, HS patients had normal left amygdala but the smallest left hippocampi; conversely, HSAS patients had larger, though still abnormal, left hippocampi and abnormal left amygdala.

Recognition accuracy was calculated for each response type (Rn, Re, Kn, Ke, Fn, Fe, see Fig. 1). Repeated-measures ANOVA with emotionality as a within-subjects factor and group as a between-subjects factor revealed no significant effect of group for either R or K responses ( $p$ greater than 0.025), but a significant effect of group for $F$ responses $(p<0.001)$, reflecting better identification of "new" stimuli by normal subjects (see Fig. 1). Hence, in the imaging analysis of subsequent memory, to best match performance in the patient and normal groups, the contrast $\mathrm{R}$ minus $\mathrm{K}$ was examined. There was no significant interaction between recognition accuracy, group, and emotionality for any response type.

We first examined the main effect (summarised in Table 1) of subsequent memory ( $R$ minus $K$, collapsed across emotionality). In the normal control group alone this contrast revealed a single MTL focus, within left hippocampus, significant at $p<0.05$ uncorrected (Fig. 2a). Note that at the same voxel, HSAS patients, with relatively larger left hippocampi, showed left hippocampal activity similar to that of the normal subjects, but HS patients, with smaller left hippocampi, showed no activity at this voxel. In the combined patient groups, four regions reached significance for the subsequent memory effect at $p<0.05$ corrected (Fig. 2): a region close to left hippocampus, possibly in the depths of collateral sulcus with similar activity across all three groups
(Fig. 2b); right hippocampus, in a position symmetrical with the left hippocampal region seen in normals, showing a larger effect in both patient groups compared to normals (Fig. 2c); right parahippocampal gyrus (Fig. 2d); and right amygdala (Fig. 2e).

The two-way interaction between subsequent memory and group revealed greater activity in right parahippocampal gyrus in the combined patient groups compared with normal controls at $p<0.05$ corrected (Fig. 3a). At the location of the right hippocampal peak showing a main effect of memory in the patients $(26,-14,-18$, Fig. 2c), there was a two-way interaction between subsequent memory effect and group, revealing greater activity in patients compared to normals at $p<0.05$ uncorrected. There were no regions showing greater activity in the normals compared to patients. Both hippocampi showed a two-way interaction between subsequent memory and emotionality, collapsed across group at $p<0.05$ corrected (Figs. 3b and $3 c)$. This effect was characterised by greater activity to emotional compared to neutral subsequently remembered stimuli. There were no regions showing greater activity to neutral items than to emotional items. Finally, the three-way interaction between memory, group (HS versus HSAS), and emotionality showed effects in an anterolateral region close to right amygdala and at the right amygdalohippocampal junction at $p<0.05$ corrected; both regions were characterised by relatively greater activity for emotional items than for neutral items in HSAS patients compared with HS patients (Fig. 4). There were no other significant three-way interactions.

In a subsequent analysis, to test specifically for hemispheric lateralisation, the peak voxel values for the main effect of memory (R minus $\mathrm{K})$ in left $(-32,-18,-18)$ and right hippocampus $(26,-14,-18)$ for all subjects were entered into a repeated-measures ANOVA with "hemisphere" (left or right) as a within-subjects effect and "group" (normals or patients) as a between-subjects effect. There was a significant interaction $(p=0.046)$ between hemisphere and group, explained by less activity in the right hippocampus in normals compared to patients. 

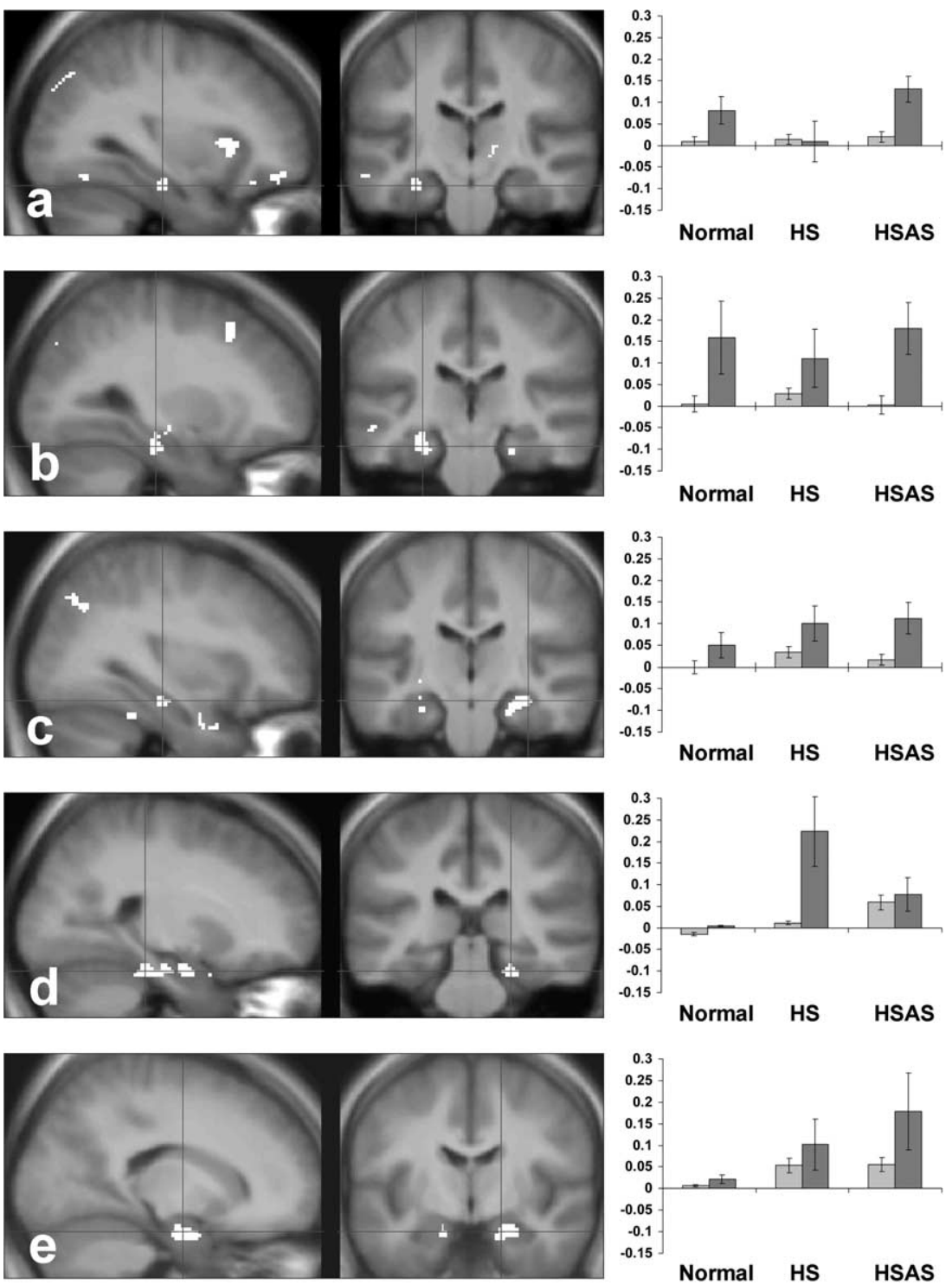

Fig. 2. Main effects of R minus K. Significant regions (all voxels $p<0.01$ in this and all subsequent figures) are superimposed onto an "average patient" T1 image, the voxel-by-voxel mean of the volumetrically normalised T1 images of all 24 patients. (a) Normal group left hippocampal peak. Although the statistical test pertains only to the main effect of memory ( $\mathrm{R}$ minus $\mathrm{K}$ ) and only to the normal subjects, parameter estimates are presented for all three groups and separately for neutral and emotional items in the bar chart. The $y$ axis represents activity in arbitrary units on this and all subsequent figures. Each bar represents the mean R minus K parameter estimate difference and the error bar \pm 1 SEM. Pale grey bars: neutral items. Dark grey bars: emotional items. (b) Combined patient groups, peak inferior to left hippocampus. Again, although the statistical test pertains only to the main effect of memory and only to the patients, parameter estimates are presented for all three groups and separately for neutral and emotional items in the bar chart. (c) Combined patient groups, right hippocampal peak. (d) Combined patient groups, right parahippocampal peak. (e) Combined patient groups, right amygdala peak.

\section{Discussion}

In nonamnesic patients with left MTL pathology we demonstrate reorganisation or reallocation of encoding processes to right MTL in two distinct ways: first, subjects with left hippocampal sclerosis (HS and HSAS groups combined) showed greater activity in right hippocampus and parahippocampal gyrus than normal subjects during successful encoding of words, compared to normal subjects. Second, subjects with left amygdala sclerosis (HSAS group) showed greater activity in the right amygdala compared to subjects without amygdala sclerosis (HS group) for successfully encoded emotional compared to neutral words. Hence, in these nonamnesic subjects with MTL pathology, verbal memory for both neutral and emotional items is associated with reallocation of encoding-related activity from leftsided MTL structures to equivalent structures in the right MTL.

Our study has a number of methodological strengths which enable a high degree of confidence in these findings. First, we examined a relatively large and highly homogeneous cohort of right-handed patients with clearly charac- 

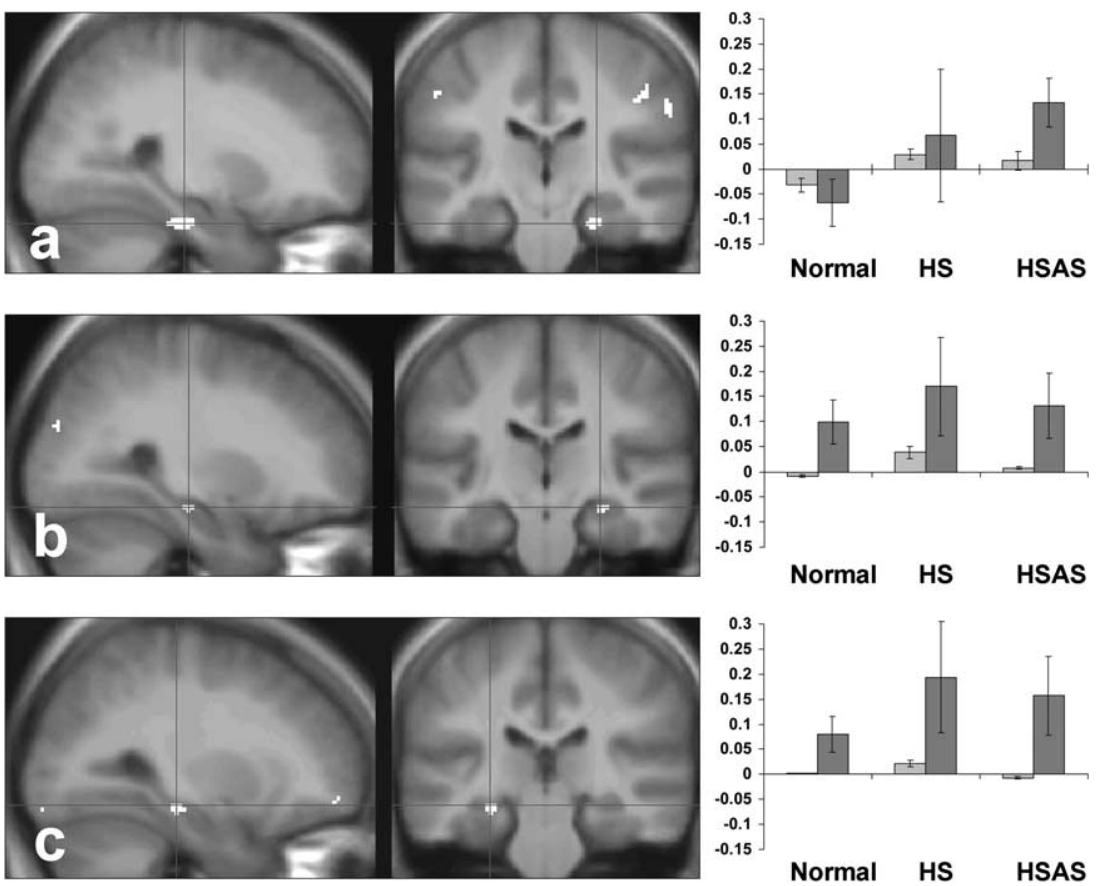

Fig. 3. Two-way interactions. (a) Interaction between main effect of memory and group (HS and HSAS combined versus normals): right parahippocampal peak. (b) Interaction between memory and emotionality (collapsed across all three groups): right hippocampus. (c) Interaction between memory and emotionality (collapsed across all three groups): left hippocampus.

terised MTL pathology. Second, we employed a psychological task which allowed good matching of task performance between normals and patients in conjunction with an eventrelated fMRI design which isolated the successful encoding processes of interest. Third, the encoding session during scanning included words with a highly emotional content, which led to robust engagement of MTL. Fourth, we employed a highly stringent, robust, and conservative statistical approach (multilevel modelling and "random effects") which permits inference to be drawn about the population
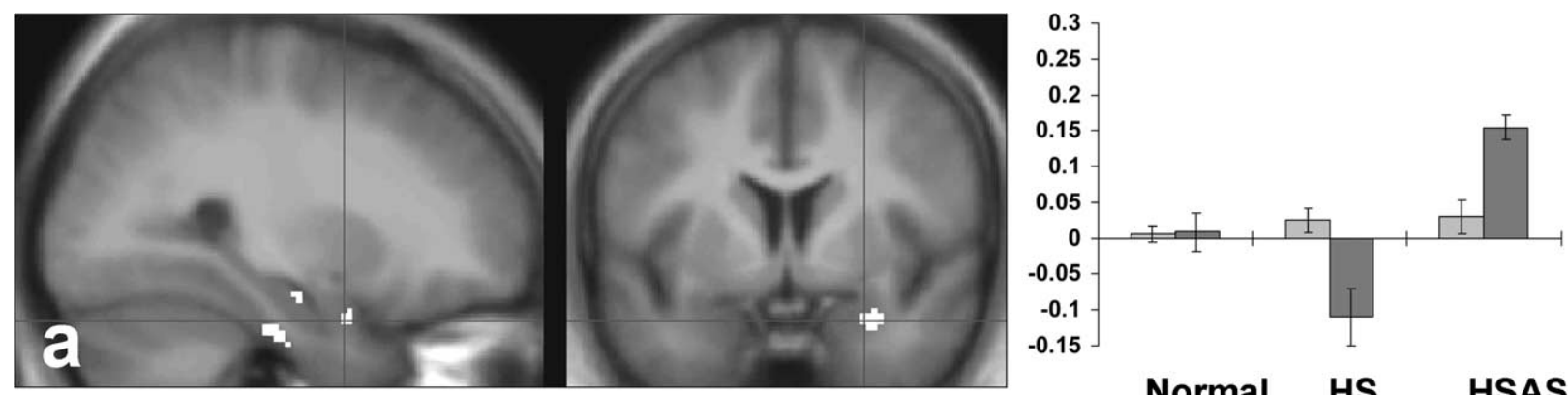

Normal HS

HSAS
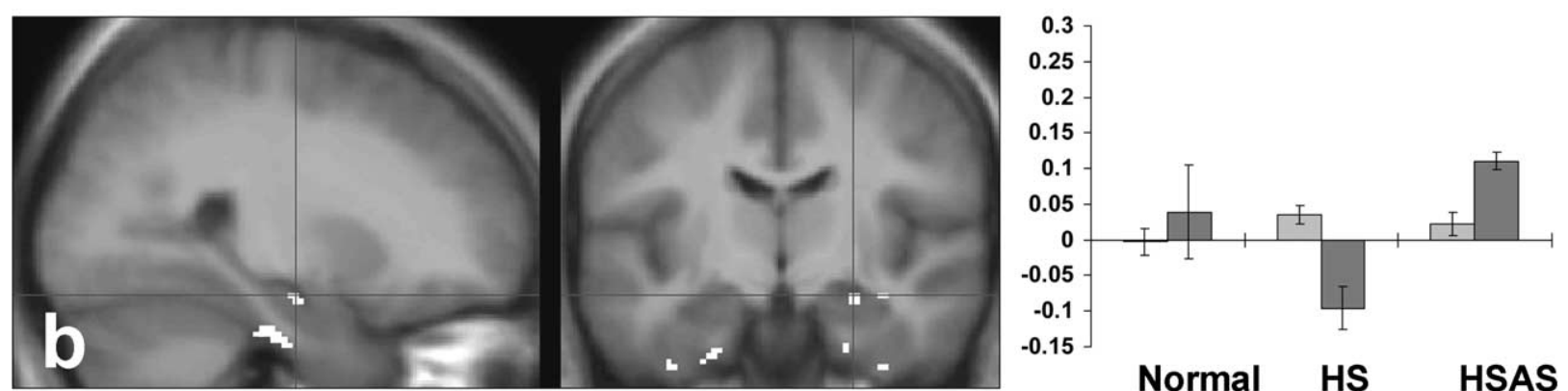

Fig. 4. Three-way interactions. (a) Interaction between memory, emotionality, and group (HSAS versus HS): right periamygdalar region. (b) Interaction between memory, emotionality, and group (HSAS versus HS): right amygdalohippocampal junction. 
from which subjects are drawn, rather than merely inference pertaining only to the subjects studied.

There have been several previous attempts to identify the site of residual memory function in subjects with left TLE. Subjects with left TLE showed less left MTL activity than subjects with right TLE during a block-design verbal task designed to identify regions engaged by semantic language decisions (Bellgowan et al., 1998); the MTL activity was assumed to be "incidental encoding," but this inference is uncertain. A parametrically analysed block-design task in seven left-TLE subjects, involving learning lists of words during one scanning session and performing covert recall during a subsequent session, revealed posterior parahippocampal activations (Talairach coordinate system $y=$ -45) during retrieval and no activations during encoding; the right side showed stronger activation than the left (Dupont et al., 2000). This very posterior region is left in situ following temporal lobe surgery for HS and would not usually be affected by lesions causing amnesia-for example, this part of the brain remains intact in HM (Corkin et al., 1997). Furthermore, in this previous study (Dupont et al., 2000) the performances of patients and controls were very significantly different $(p<0.0007)$ and the patients did not show greater activation of any MTL region compared to controls. In a group of six left-TLE patients, a block-design study involving presentation of novel or familiar words revealed that novel words engaged the right MTL but no direct comparison was made to normal controls and memory was not tested (Golby et al., 2002). Covert recall of familiar routes between places in a familiar town, compared with covert counting in a block design, revealed symmetric MTL activation of uncertain location in normal subjects and similar, but asymmetric, MTL activation in TLE patients favouring the unaffected MTL (Jokeit et al., 2001). Similarly, presentation of complex visual scenes (assumed to result in incidental encoding) compared to "visual noise" patterns in a block design resulted in asymmetric MTL activation in TLE patients (Detre et al., 1998). None of these prior studies have used a design which permits memory to be directly examined and none has revealed reorganisation of verbal memory in comparison with controls. Although one previous study showed a difference in the extent and magnitude of activation in the left hippocampus during a memory task between normal subjects and carriers of a risk gene for Alzheimer's disease (Bookheimer et al., 2000), there are no studies of which we are aware that have identified reorganisation of verbal memory to another anatomical location.

Epilepsy is an important public health problem and HS in particular is the most common cause of drug-resistant epilepsy. A recent randomised study of temporal lobe resection in such subjects (Wiebe et al., 2001) has shown resection of the affected temporal lobe to be superior to drug treatment for controlling seizures. Avoidance of postoperative memory impairment is a crucial objective in this setting (Bax- endale, 1998). Although psychometric tests, structural imaging, and the Wada test may identify patients at special risk of postoperative amnesia, a need exists for functional mapping of residual memory abilities. The growth in understanding of the functional anatomy of memory in normal subjects has not been matched by knowledge concerning how the human brain compensates following injury to primary memory regions. Our study suggests that effective verbal memory encoding in subjects with left hippocampal and hippocampal plus amygdala damage is mediated by recruitment of parallel or redundant memory systems within the right MTL.

\section{Acknowledgments}

We thank Professor Ley Sander and Drs. Sanjay Sisodiya, Hannah Cock, Shelagh Smith, and Matthias Koepp for referring patients to the study. M.P.R. is supported by a fellowship of the Medical Research Council, UK. R.J.D. is supported by a Wellcome Trust Programme Grant.

\section{References}

Adolphs, R., Cahill, L., Schul, R., Babinsky, R., 1997. Impaired declarative memory for emotional material following bilateral amygdala damage in humans. Learn. Memory 4, 291-300.

Bartlett, P.A., Richardson, M.P., Duncan, J.S., 2002. Measurement of amygdala T2 relaxation time in temporal lobe epilepsy. J. Neurol. Neurosurg. Psychiatry 73, 753-735.

Baxendale, S.A., 1998. Amnesia in temporal lobectomy patients: historical perspective and review. Seizure 7, 15-24.

Bellgowan, P.S., Binder, J.R., Swanson, S.J., Hammeke, T.A., Springer, J.A., Frost, J.A., Mueller, W.M., Morris, G.L., 1998. Side of seizure focus predicts left medial temporal lobe activation during verbal encoding. Neurology 51, 479-484.

Blasi, V., Young, A.C., Tansy, A.P., Petersen, S.E., Snyder, A.Z., Corbetta, M., 2002. Word retrieval learning modulates right frontal cortex in patients with left frontal damage. Neuron 36, 159-170.

Bookheimer, S.Y., Strojwas, M.H., Cohen, M.S., Saunders, A.M., PericakVance, M.A., Mazziotta, J.C., Small, G.W., 2000. Patterns of brain activation in people at risk for Alzheimer's disease. N. Engl. J. Med. 343, 450-456.

Cabeza, R., Nyberg, L., 2000. Imaging cognition. II. An empirical review of 275 PET and fMRI studies. J. Cognit. Neurosci. 12, 1-47.

Corkin, S., Amaral, D.G., Gonzalez, R.G., Johnson, K.A., Hyman, B.T., 1997. H.M.'s medial temporal lobe lesion: findings from magnetic resonance imaging. J. Neurosci. 17, 3964-3979.

Detre, J.A., Maccotta, L., King, D., Alsop, D.C., Glosser, G., D’Esposito, M., Zarahn, E., Aguirre, G.K., French, J.A., 1998. Functional MRI lateralization of memory in temporal lobe epilepsy. Neurology 50, 926-932.

Dolan, R.J., 2002. Emotion, cognition and behaviour. Science 298, 11911194.

Duncan, J.S., 1997. Imaging and epilepsy. Brain 120, 376-389.

Duncan, J.S., Bartlett, P., Barker, G.J., 1996. Technique for measuring hippocampal T2 relaxation time. Am. J. Neuroradiol. 17, 1805-1810.

Dupont, S., Van de Moortele, P.F., Samson, S., Hasboun, D., Poline, J.B., Adam, C., Lehericy, S., Le Bihan, D., Samson, Y., Baulac, M., 2000. Episodic memory in left temporal lobe epilepsy: a functional MRI study. Brain 123, 1722-1732. 
Frisk, V., Milner, B., 1990. The role of the left hippocampal region in the acquisition and retention of story content. Neuropsychologia 28, 349359.

Friston, K.J., Fletcher, P., Josephs, O., Holmes, A., Rugg, M.D., Turner, R., 1998. Event-related fMRI: characterizing differential responses. NeuroImage 7, 30-40.

Friston, K.J., Holmes, A.P., Worsley, K.J., Poline, J.B., Frith, C.D., Frackowiak, R.S.J., 1995. Statistical parametric maps in functional imaging: a general linear approach. Hum. Brain Mapp. 2, 189-210.

Golby, A.J., Poldrack, R.A., Illes, J., Chen, D., Desmond, J.E., Gabrieli, J.D., 2002. Memory lateralisation in medial temporal lobe epilepsy assessed by functional MRI. Epilepsia 43, 855-863.

Hamann, S.B., Ely, T.D., Grafton, S.T., Kilts, C.D., 1999. Amygdala activity related to enhanced memory for pleasant and aversive stimuli. Nat. Neurosci. 2, 289-293.

Helmstaedter, C., Kurthen, M., 2001. Memory and epilepsy: characteristics, course, and influence of drugs and surgery. Curr. Opin. Neurol. 14, 211-216.

Hudson, L.P., Munoz, D.G., Miller, L., McLachlan, R.S., Girvin, J.P. Blume, W.T., 1993. Amygdaloid sclerosis in temporal lobe epilepsy. Ann. Neurol. 33, 622-631.

Johansen-Berg, H., Dawes, H., Guy, C., Smith, S.M., Wade, D.T., Matthews, P.M., 2002. Correlation between motor improvements and altered fMRI activity after rehabilitative therapy. Brain 125, 2731-2742.

Jokeit, H., Okujava, M., Woermann, F.G., 2001. Memory fMRI lateralizes temporal lobe epilepsy. Neurology 57, 1786-1793.

Kelley, W.M., Miezin, F.M., McDermott, K.B., Buckner, R.L., Raichle, M.E., Cohen, N.J., Ollinger, J.M., Akbudak, E., Conturo, T.E., Snyder, A.Z., Petersen, S.E., 1998. Hemispheric specialization in human dorsa frontal cortex and medial temporal lobe for verbal and nonverbal memory encoding. Neuron 20, 927-936.

Lepage, M., Habib, R., Tulving, E., 1998. Hippocampal PET activations of memory encoding and retrieval: the HIPER model. Hippocampus 8 , 313-322.

Neville, H.J., Bavelier, D., Corina, D., Rauschecker, J., Karni, A., Lalwani, A., Braun, A., Clark, V., Jezzard, P., Turner, R., 1998. Cerebral organization for language in deaf and hearing subjects: biological constraints and effects of experience. Proc. Natl. Acad. Sci. USA 95, 922-929.

Otten, L.J., Henson, R.N., Rugg, M.D., 2001. Depth of processing effects on neural correlates of memory encoding: relationship between findings from across- and within-task comparisons. Brain 124, 399-412.
Phelps, E.A., LaBar, K.S., Spencer, D.D., 1997. Memory for emotional words following unilateral temporal lobectomy. Brain Cognit. 35, 85109 .

Pineiro, R., Pendlebury, S., Johansen-Berg, H., Matthews, P.M., 2001. Functional MRI detects posterior shifts in primary sensorimotor cortex activation after stroke: evidence of local adaptive reorganization? Stroke 32, 1134-1139.

Sadato, N., Pascual-Leone, A., Grafman, J., Ibanez, V., Deiber, M.P., Dold, G., Hallett, M., 1996. Activation of the primary visual cortex by Braille reading in blind subjects. Nature $380,526-528$.

Scoville, W.B., Milner, B., 1957. Loss of recent memory after bilateral hippocampal lesions. J. Neurosurg. Psychiatry 20, 11-21.

Sisodiya, S.M., Moran, N., Free, S.L., Kitchen, N.D., Stevens, J.M., Harkness, W.F., Fish, D.R., Shorvon, S.D., 1997. Correlation of widespread preoperative magnetic resonance imaging changes with unsuccessful surgery for hippocampal sclerosis. Ann. Neurol. 41, 490-496.

Smith, M.L., Milner, B., 1981. The role of the right hippocampus in the recall of spatial location. Neuropsychologia 19, 781-793.

Squire, L.R., 1992. Memory and the hippocampus: a synthesis from findings in rats, monkeys and humans. Psychol. Rev. 99, 195-231.

Squire, L.R., Zola-Morgan, S., 1991. The medial temporal lobe memory system. Science 253, 1380-1386.

Staudt, M., Grodd, W., Gerloff, C., Erb, M., Stitz, J., Krageloh-Mann, I., 2002. Two types of ipsilateral reorganization in congenital hemiparesis: a TMS and fMRI study. Brain 125, 2222-2237.

Strange, B.A., Henson, R.N., Friston, K.J., Dolan, R.J., 2000. Brain mechanisms for detecting perceptual, semantic, and emotional deviance. NeuroImage 12, 425-433.

Tulving, E., 1985. Memory and consciousness. Can. Psychol. 26, 1-12.

Van Paesschen, W., Connelly, A., Duncan, J.S., 1996. The amygdala and intractable temporal lobe epilepsy: a quantitative magnetic resonance imaging study. Neurology 47, 1021-1031.

Warburton, E., Price, C.J., Swinburn, K., Wise, R.J., 1999. Mechanisms of recovery from aphasia: evidence from positron emission tomography studies. J. Neurol. Neurosurg. Psychiatry 66, 155-161.

Wiebe, S., Blume, W.T., Girvin, J.P., and Eliasziw, M., 2001. A randomized, controlled trial of surgery for temporal-lobe epilepsy. N. Engl. J. Med. 345, 311-318.

Woermann, F.G., Barker, G.J., Birnie, K.D., Meencke, H.J., Duncan, J.S., 1998. Regional changes in hippocampal T2 relaxation and volume: a quantitative magnetic resonance imaging study of hippocampal sclerosis. J. Neurol. Neurosurg. Psychiatry 65, 656-664. 